\title{
CLASIFICACIÓN DE BOSQUES SOBRE ARENA BLANCA DE LA ZONA RESERVADA ALLPAHUAYO-MISHANA
}

\author{
Roosevelt García Villacorta ${ }^{1}$, Manuel Ahuite Reáteguii ${ }^{1}$ y Mauro Olórtegui Zumaeta ${ }^{1}$
}

\begin{abstract}
RESUMEN
La Zona Reservada Allpahuayo-Mishana (ZRAM) alberga las áreas más extensas de bosques sobre arena blanca, localmente conocidos como varillales, protegidas actualmente en el Perú. Con el propósito de obtener información que ayude en el manejo y la conservación de este tipo de vegetación, nosotros estudiamos la composición florística, estructura y características ambientales de 44 parcelas, 20 × $20 \mathrm{~m}$, ubicadas en los suelos de arena blanca de la ZRAM, Loreto, Perú ( $3^{\circ} 53^{\prime}$ S, $73^{\circ} 25^{\prime}$ O, 110-180 m.s.n.m.). Para la selección de los lugares de muestreo en el área utilizamos una imagen de satélite Landsat TM 5. Seleccionamos los lugares de muestreo, tratando de abarcar la mayor variación posible que podíamos observar en los colores de la imagen de satélite, con el fin de muestrear varillales potencialmente diferentes en el campo. Las parcelas estuvieron ubicadas a lo largo de transectos que atravesaron parches heterogéneos de varillal. En cada parcela registramos, en cinco clases diamétricas, a todos los individuos que tenían al menos un metro de altura e hicimos una estimación de la cobertura herbácea. También medimos la altura del dosel y grosor de la capa orgánica. Realizamos análisis de agrupamiento (cluster analysis), entre las parcelas, usando como variables el número de tallos por parcela, la altura del dosel y la calidad del drenaje expresada por el grosor de la capa orgánica (capa orgánica delgada = buen drenaje, y viceversa). Utilizamos además la abundancia y frecuencia de las especies en los grupos para definir especies indicadoras (indicator species analysis). En el contexto de nuestro estudio, y utilizando la terminología regional para estos bosques, reconocemos la existencia de cinco tipos de varillales en la ZRAM: varillal alto seco, varillal alto húmedo, varillal bajo seco, varillal bajo húmedo y chamizal.
\end{abstract}

Palabras clave: Allpahuayo-Mishana, Amazonía peruana, análisis de agrupamiento jerárquico, bosques sobre arena blanca, clasificación de bosques, especies indicadoras, varillales.

\begin{abstract}
The Allpahuayo-Mishana Reserved Zone (ZRAM) is home to the most extensive areas of forest on white sand (known locally as varillales) currently under protection in Peru. In order to obtain data that will help the management and conservation of this type of vegetation we studied the structure, floristic composition and environmental characteristics of 44 plots measuring $20 \mathrm{~m}$ x $20 \mathrm{~m}$, located in the white sand soils of the ZRAM, in Loreto, Peru ( $3^{\circ} 53^{\prime} \mathrm{S}, 7^{\circ} 25^{\prime} \mathrm{O}, 110-180 \mathrm{~m}$ a.s.l.). We used Landsat TM 5 satellite images to select the sample areas, trying to include the widest possible variation of colours observed in the satellite images, so as to show potentially different varillales in the area. The plots were placed along transects traversing heterogeneous patches of varillal. In each plot we recorded all the individual stems with a height of at least $1 \mathrm{~m}$ in five diameter classes, and estimated the cover of herbaceous plants. We also evaluated the height of the canopy and thickness of the organic layer. We did a cluster analysis of the plots using the variables of stem number, canopy height and drainage quality expressed by the thickness of the organic layer (thin organic layer $=$ good drainage, and vice versa). In addition, we used the abundance and frequency of the species in each group to define their indicator values. In the context of our study, using regional terminology for these forests, we recognized the existence of five types of forests on white sand (varillal) in the ZRAM: high-dry-open white sand forest (varillal alto seco), high-wet-open white sand forest (varillal alto húmedo), low-dry-dense white sand forest (varillal bajo seco), low-wet-open white sand forest (varillal bajo húmedo) and very low-wet-very dense white sand forest (chamizal).
\end{abstract}

Key words: Allpahuayo-Mishana, cluster analysis, forest classification, indicator species, Peruvian Amazon, white-sand forests.

1 Proyecto Diversidad Biológica de la Amazonía Peruana - BIODAMAZ, Perú - Finlandia. Av. Abelardo Quiñones, km 2.5, Iquitos, Perú. Correo electrónico: biodamaz@iiap.org.pe 


\section{INTRODUCCIÓN}

Los suelos de arena blanca se encuentran distribuidos en forma dispersa en varios lugares de la cuenca amazónica. Asociada con estos suelos, extremadamente pobres en nutrientes, y restringida a ellos (Vitousek y Sanford, 1986; Coomes, 1997; Kauffman et al., 1998), crece una vegetación muy particular, caracterizada por tener especies monodominantes, baja diversidad y elevado endemismo. La fisonomía de estos bosques puede variar desde bosques enanos con alta cobertura herbácea y elevada densidad de tallos, hasta bosques altos con dosel cerrado y reducida cobertura herbácea. Se ha sugerido que las condiciones del drenaje, controlado por la presencia superficial o profunda de una capa impermeable debajo del sustrato de arena (Ruokolainen y Tuomisto, 1998), las condiciones de elevada acidez para la germinación de las semillas (Proctor, 1998), o ambos factores (Duivenvoorden, 1996), serían responsables de esta variación en su estructura.

En la Amazonía peruana estos bosques reciben el nombre de varillales, por su relativa alta densidad de tallos verticales. El área más grande de varillales, conocida en Perú, se encuentra hacia el suroeste de la ciudad de Iquitos y a lo largo de la margen derecha del río Nanay, donde éstos están distribuidos en parches de distintos tamaños. Estudios recientes de geología en el área sugieren que estos suelos, al menos en esta parte de la Amazonía, tienen un origen fluvial (M. Räsänen, com. pers.). Al parecer un río de aguas negras, un protoNanay, pasaba por los lugares donde ahora están ubicadas las manchas de arena en el área de Iquitos, y fue migrando hacia el oeste, dejando terrazas de arena blanca de cuarzo, que fueron ocupadas por una vegetación adaptada a este tipo de sustrato. También se ha reportado la existencia de extensiones más reducidas de varillales en Jenaro Herrera (río Ucayali), los alrededores de Yurimaguas (Encarnación, 1993), bajo río Morona, las cercanías de Jeberos (bajo río Huallaga) y Tamshiyacu (Álvarez, 2002; P. Fine, com. pers.), entre otros lugares.

En un contexto geográfico más amplio, parches de vegetación similar con nombres variables también ocurren en las cuencas del alto río Negro y río Blanco, en el norte de Brasil (campina, campinarana, caatinga amazónica), suroeste de Venezuela (caatinga, bana), Guyanas (wallaba), y este de Colombia, y son equivalentes a los kerengas o 'heath forests' de los trópicos de Asia. Debido a su particularidad y relativa simplicidad, la vegetación de arena blanca en la Amazonía de Brasil, Venezuela y Guyanas ha sido tema de considerable escrutinio por los botánicos (Rodríguez, 1961; Takeuchi, 1962; Anderson et al., 1975; Klinge et al., 1977; Anderson, 1981; ter Steege et al., 1993; Coomes y Grubb, 1996, entre otros). Sin embargo, estudios sistemáticos cuantitativos de los bosques sobre arena blanca en la Amazonía occidental son escasos (Gentry, 1988; van der Werff, 1992; Tuomisto y Ruokolainen, 1994; Duivenvoorden y Lips, 1995; Vormisto et al., 2000).

Recientemente se ha creado la Zona Reservada Allpahuayo-Mishana (ZRAM) con la intención de proteger los varillales cercanos a la ciudad de Iquitos de la expansión urbana y de la deforestación. Aunque se realizaron algunos estudios que presentaron una clasificación preliminar de estos bosques (Revilla, 1974; Encarnación, 1985; IIAP, 2000), estos se han basado principalmente en la impresión personal con respecto a su altura, condiciones de drenaje y en el conocimiento empírico de algunas especies indicadoras. Sin embargo, de acuerdo a nuestro conocimiento nadie ha documentado cuantitativamente cómo estas características pueden variar dentro de los varillales y apoyar la separación de uno o más tipos de bosques sobre arena blanca. En este artículo nosotros presentamos datos cuantitativos de la estructura, condiciones ambientales y composición florística de los varillales de la ZRAM, con la meta principal de producir una clasificación de estos bosques y proporcionar el conocimiento básico que ayude en su manejo y conservación. Este trabajo se hizo en el marco del proyecto Diversidad Biológica de la Amazonía Peruana (BIODAMAZ), Perú-Finlandia. ${ }^{2}$

2 Proyecto Diversidad Biológica de la Amazonía Peruana (BIODAMAZ) es un convenio entre los gobiernos del Perú y de Finlandia ejecutado conjuntamente por el Instituto de Investigaciones de la Amazonía Peruana (IIAP) y por el consorcio finlandés formado por la empresa de consultorías ambientales, Biota BD Oy, y por la Universidad de Turku. 


\section{MATERIAL Y MÉTODO}

\subsection{Lugares de estudio}

La Zona Reservada Allpahuayo-Mishana ( $3^{\circ} 53^{\prime}$ S S, $73^{\circ} 25^{\prime}$ O, $110-180$ m.s.n.m.) se encuentra ubicada al suroeste de Iquitos en la Amazonía peruana, entre el río Nanay en el noroeste y la carretera Iquitos - Nauta hacia el sur. El clima es cálido y húmedo (con una media de precipitación anual alrededor de $3000 \mathrm{~mm}$ y un promedio de temperatura de $26^{\circ} \mathrm{C}$; Marengo, 1998). Las condiciones edáficas son extremadamente variables, representando una combinación de suelos que varían en textura desde arcilla hasta arena de cuarzo casi pura, y en drenaje desde pantanos anegados hasta cimas de colinas bien drenadas.

Entre enero y abril del 2002 muestreamos las especies de plantas en los bosques presentes sobre las arenas blancas (varillales) de la ZRAM. Las unidades de arena blanca de Iquitos aparecen eventualmente en la imagen Landsat TM 5 como manchas oscuras de color verde, y dentro de estas manchas se puede observar partes de coloración más clara que aparentemente corresponden a chamizales, un tipo de varillal enano, ya que al parecer el varillal húmedo tiene color más oscuro que el varillal seco (Kalliola et al., 1998). Por esta razón nosotros seleccionamos los lugares de estudio tratando de ubicar las parcelas de muestreo donde hubiese la mayor variación posible en los colores de la imagen de satélite, indicando posibles diferencias en la vegetación. El muestreo tuvo lugar en 44 parcelas de 0.04 ha $(20$ x $20 \mathrm{~m})$ cada una, las cuales estuvieron situadas en varillales fisionómicamente homogéneos a lo largo de transectos que atravesaron parches heterogéneos de varillal, y con una distancia promedio de separación entre parcelas de $200 \mathrm{~m}$. Se trató de evitar claros grandes causados por deforestación.

Las primeras diez parcelas están ubicadas en el área del Instituto Nacional de Investigación Agraria (INIA) ( $3^{\circ} 57^{\prime} 08.8^{\prime \prime} \mathrm{S}, 7^{\circ} 24^{\prime} 33.7^{\prime \prime}$ O), a lo largo de la trocha donde L. Klemola (Universidad de Turku, Finlandia), realizó trabajos de campo para estudiar las capas sedimentológicas. Trece parcelas se encuentran distribuidas

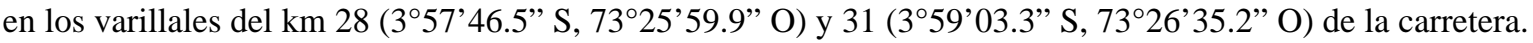
En los bosques sobre arena blanca del poblado de Yuto, río Nanay ( $\left.3^{\circ} 55^{\prime} 04.4^{\prime \prime} \mathrm{S}, 73^{\circ} 31^{\prime} 56.0^{\prime \prime} \mathrm{O}\right)$, establecimos 20 parcelas de muestreo. La parcela número $44\left(3^{\circ} 57^{\prime} 0.87^{\prime}\right.$ ' S, $73^{\circ} 25^{\prime} 50.2^{\prime}$ O O), está ubicada en el varillal más allá del lugar conocido como 'Frutales', en la Estación Biológica Allpahuayo.

Cada parcela de muestreo fue georeferenciada con GPS (Sistema de Posicionamiento Global, GARMIN, GPS 12), y marcada en las esquinas con tubos plásticos de PVC (aprox. $50 \mathrm{~cm}$ ). La parte superior de los tubos fue pintada con pintura roja, para facilitar su localización posterior. Cada unidad de muestreo constituye parcelas permanentes para futuros estudios ecológicos de flora y fauna. En la Tabla 3 presentamos un resumen con las características estructurales y ambientales de las parcelas permanentes.

\subsection{Evaluación de la vegetación}

Para facilitar el muestreo cada parcela fue subdividida en 16 subparcelas de 5 x $5 \mathrm{~m}$. En cada subparcela contamos el número total de tallos en posición vertical en las siguientes categorías: desde $1 \mathrm{~m}$ de alto hasta $1 \mathrm{~cm}$ de diámetro a la altura del pecho (DAP), $1-2.5 \mathrm{~cm}, 2.5-5 \mathrm{~cm}$ y $5-10 \mathrm{~cm}$. Medimos individualmente el DAP de aquellos individuos con más de $10 \mathrm{~cm}$ de DAP. Estimamos además la cobertura de las plantas herbáceas en el piso del bosque. Las palmeras que tenían el límite de bifurcación de las hojas mayor o igual a $1 \mathrm{~m}$ de altura fueron incluidas en el inventario. Registramos además sólo las plantas rastreras y lianas que presentaron una alta densidad de tallos en las parcelas.

La altura promedio del dosel y de los árboles emergentes se obtuvo midiendo con clinómetro un árbol representativo de ese estrato arbóreo. Medimos además el grosor de la materia orgánica en cada parcela (5 puntos por parcela). Colectamos un ejemplar de referencia por cada especie registrada en el muestreo que no estuvo presente en una colección anterior. Las colecciones botánicas (R. García 1301-2082) están depositadas en el Herbario Amazonense (AMAZ) de la Universidad Nacional de la Amazonía Peruana. Los especímenes fueron identificados usando la literatura, la base de datos Tropicos (www.mobot.org), la base de datos Cassia 
(www.nybg.org), por comparación en el herbario y consulta a especialistas. Especies no identificadas fueron categorizadas como morfoespecies en el nivel en el cual fueron identificadas, por ejemplo, Lauraceae sp. 1, Styrax sp. 1. Los taxa identificados al nivel de familia siguen a Judd et al. (1999), y están listados en la Tabla 2.

\subsection{Análisis de agrupamiento jerárquico}

Antes de ejecutar el análisis de agrupamiento normalizamos los datos de las tres variables (Tabla 3), de modo tal que todos tuvieran como promedio 0 y varianza 1. Esto es necesario porque queríamos asegurarnos de que todas las variables tuvieran el mismo nivel de importancia al realizar la clasificación.

Un análisis previo de correlación del número de tallos por parcela entre las cinco clases diamétricas mostró una fuerte correlación entre las cuatro primeras clases. El número de tallos por parcela en la clase $>10 \mathrm{~cm}$ de DAP fue muy variable. Por esta razón decidimos juntar las cuatro primeras clases en una sola (de $1 \mathrm{~m}$ de altura - 10 $\mathrm{cm}$ de DAP). Sacamos del análisis a los tallos mayores de $10 \mathrm{~cm}$ de DAP porque el tamaño de la parcela nos pareció pequeño para muestrear adecuadamente los tallos grandes, y además están correlacionados con la altura del dosel.

Ejecutamos análisis de agrupamiento (cluster analysis) entre las 44 parcelas, usando como variables el número total de tallos de $1 \mathrm{~m}$ de altura hasta $10 \mathrm{~cm}$ de DAP presentes en cada parcela, la altura del dosel y la calidad del drenaje, representada por el grosor de la materia orgánica (capa fina de materia orgánica = buen drenaje y viceversa). El propósito del análisis de agrupamiento es definir grupos de parcelas sobre la base de sus similitudes con respecto a las variables. El método de enlace entre los grupos fue la mediana de la distancia euclidiana con respecto a otro grupo particular. En la clasificación por la mediana, después de la formación de un nuevo grupo, se calcula una distancia mediana desde este grupo a todos los otros grupos (o parcelas solitarias), sobre la base de las distancias de los enlaces que este grupo tiene con los otros. La próxima unión se hace en la distancia mediana más corta entre los grupos (Legendre y Legendre, 1998).

Después de realizar el análisis de agrupamiento seguimos algunos criterios (cf. Tabla 3), para definir los grupos de parcelas formados y que representarían tipos de varillales diferentes. Estos criterios fueron: alrededor de 1000 tallos = varillal libre; de 1000 a 2000 tallos = varillal denso; y más de 2000 tallos = varillal muy denso; altura menor a $5 \mathrm{~m}=$ varillal muy bajo; de $5-15 \mathrm{~m}=$ varillal bajo; mayor de $15 \mathrm{~m}=$ varillal alto; hasta más o menos $11 \mathrm{~cm}$ de materia orgánica = varillal seco; más de $11 \mathrm{~cm}$ de materia orgánica = varillal húmedo.

\subsection{Análisis de especies indicadoras}

Utilizamos además la información sobre la concentración de la abundancia de las especies en un grupo particular, y la fidelidad o frecuencia de ocurrencia de una especie en un grupo particular, para detectar y describir el valor de las diferentes especies que pueden indicar condiciones ambientales entre los grupos obtenidos (análisis de especies indicadoras, Dufrêne y Legendre, 1997). Usamos la prueba de Monte Carlo (Legendre y Legendre, 1998), con 10000 aleatorizaciones para evaluar la significancia estadística del valor indicador máximo (maximum indicator value), para cada especie. Asumimos que si una especie dada tiene un valor $p$ por debajo de 0.05 (5\%) para un grupo, existe poca probabilidad de que esté presente en otro grupo en la misma concentración de abundancia, y por lo tanto, es un buen indicador de ese tipo de varillal. En otras palabras, si al azar una especie dada puede alcanzar un valor mayor al $5 \%$ dentro de un grupo particular, probablemente esa especie no responde a las variables que formaron ese grupo y por lo tanto no es buen indicador.

Todos los análisis numéricos se ejecutaron con el paquete estadístico para análisis multivariado PC-ORD, versión 4 (McCune y Mefford, 1999). 


\section{RESULTADOS}

La Figura 1 muestra el resultado del análisis de agrupamiento y los grupos formados. Al utilizar los criterios para definir los tipos de varillales según sus características obtuvimos cinco tipos, que se muestran en la Tabla 1. Los varillales extremos (altos secos y los muy bajos y húmedos o chamizales), presentaron el mayor número de especies indicadoras, mientras que el varillal bajo-húmedo-libre (varillal bajo húmedo) y el varillal bajoseco-denso (varillal bajo seco) solo presentaron una especie indicadora: Virola pavonis y Dicymbe uaiparuensis, respectivamente.

En la Tabla 2 presentamos también los resultados del análisis de especies indicadoras con sus respectivos niveles de significancia según la prueba de Monte Carlo. De las 298 especies encontradas durante el estudio, solo 29 especies mostraron suficiente consistencia en su presencia y abundancia para permitir que sean identificadas como potenciales especies indicadoras de algún tipo de varillal (Tabla 1).

Figura 1. Análisis de agrupamiento según densidad de tallos, drenaje y dosel.

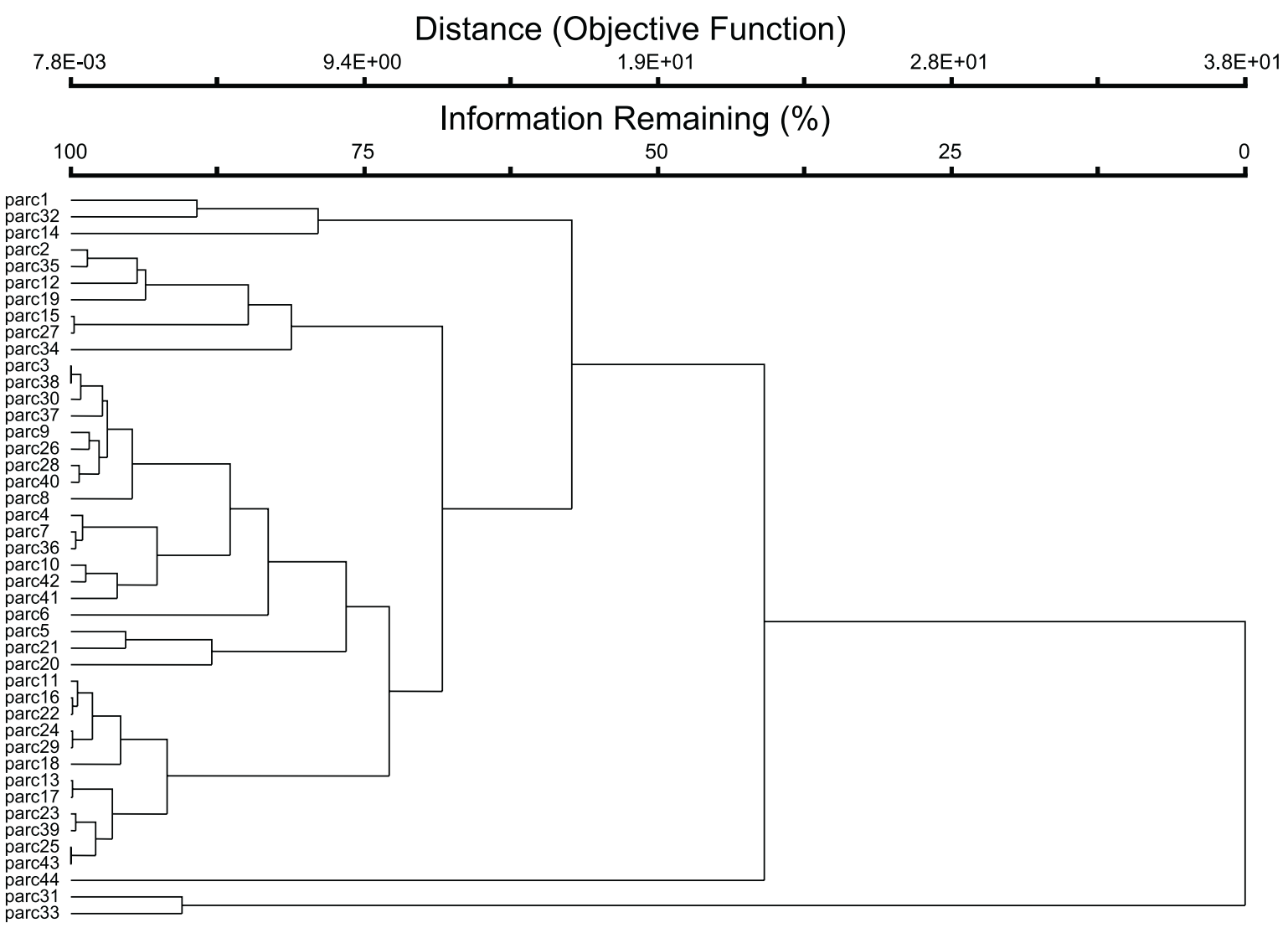


Tabla 1. Tipos de varillales según características de altura, drenaje y densidad de tallos con las parcelas y sus especies indicadoras representativas. Los números de las parcelas corresponden a los números correlativos asignados a las parcelas durante el muestreo.

\begin{tabular}{|c|c|c|}
\hline Tipo de varillal & Parcelas & Especies indicadoras \\
\hline $\begin{array}{l}\text { GRUPO 1: } 5-15 \mathrm{~m} \text { de altura, mayor de } 11 \mathrm{~cm} \\
\text { de materia orgánica y ca. de } 1000 \text { tallos } \\
\text { Varillal bajo-húmedo-libre } \\
\text { (varillal bajo húmedo) }\end{array}$ & 1,32 y 14 & Virola pavonis \\
\hline $\begin{array}{l}\text { GRUPO 2: } 5-15 \mathrm{~m} \text { de altura, } 0-11 \mathrm{~cm} \text { de } \\
\text { materia orgánica y } 1000 \text { a } 2000 \text { tallos } \\
\text { Varillal bajo-seco-denso } \\
\text { (varillal bajo seco) }\end{array}$ & $2,35,12,19,15,27$ у 34 & Dicymbe uaiparuensis \\
\hline $\begin{array}{l}\text { GRUPO 3: mayor de } 15 \mathrm{~m} \text { de altura, mayor } \\
\text { de } 11 \mathrm{~cm} \text { de materia orgánica y ca. de } 1000 \\
\text { tallos } \\
\text { Varillal alto-húmedo-libre } \\
\text { (varillal alto húmedo) }\end{array}$ & $\begin{array}{l}3,38,30,37,9,26,28 \\
40,8,4,7,36,10,42,41 \\
6,5,21 \text { у } 20\end{array}$ & $\begin{array}{l}\text { Adiscanthus fusciflorus, } \\
\text { Chrysophyllum manaosensis, } \\
\text { Styrax sp. } 1\end{array}$ \\
\hline $\begin{array}{l}\text { GRUPO 4: mayor de } 15 \mathrm{~m} \text { altura, } 0-11 \mathrm{~cm} \text { de } \\
\text { materia orgánica y ca. de } 1000 \text { tallos } \\
\text { Varillal alto-seco-libre } \\
\text { (varillal alto seco) }\end{array}$ & $\begin{array}{l}11,16,22,24,29,18,13 \\
17,23,39,25,43 \text { у } 44\end{array}$ & $\begin{array}{l}\text { Oxandra euneura, Aspidosperma } \\
\text { pichonianum, Buchenavia } \\
\text { reticulata, Couepia parillo, } \\
\text { Aparishtmium cordatum, Mabea } \\
\text { subsessilis, Pausandra martinii, } \\
\text { Byrsonima stipulina, Myrtaceae sp. } \\
\text { 2, Myrtaceae sp. } 4 \text {, Matayba sp. } 1 \text {, } \\
\text { Simaba polyphylla }\end{array}$ \\
\hline $\begin{array}{l}\text { GRUPO 5: menor a } 5 \mathrm{~m} \text { altura, mayor de } 11 \\
\mathrm{~cm} \text { de materia orgánica y mayor de } 2000 \\
\text { tallos } \\
\text { Varillal muy bajo-húmedo-muy denso } \\
\text { (chamizal) }\end{array}$ & 31 y 33 & $\begin{array}{l}\text { Dendropanax umbellatus, } \\
\text { Doliocarpus dentatus, Sloanea } \\
\text { spathulata, Graffenrieda limbata, } \\
\text { Neea divaricata, Epistephium } \\
\text { parviflorum, Trichomanes } \\
\text { martiusii, Psychotria sp. } 4, \\
\text { Rubiaceae sp. } 1, \text { Siparuna } \\
\text { guianensis, Anacardium giganteum }\end{array}$ \\
\hline
\end{tabular}

\section{DISCUSIÓN}

Aunque la separación en cinco tipos de varillales parece conveniente y puede ser utilizada como esquema para distinguirlos entre sí, también se presentaron algunas características a ser tomadas en cuenta: Los varillales altos (secos y húmedos) agruparon muchas más parcelas (32 parcelas), que los varillales bajos (10 parcelas), y muy bajos ( 2 parcelas). Al parecer esos varillales (altos secos y altos húmedos) son más comunes en los suelos de arena blanca de la ZRAM, ya que fue posible encontrarlos con más frecuencia durante el muestreo en distintos lugares (Tabla 1). Estos resultados son más consistentes si consideramos que para la selección de las áreas de estudio tomamos en cuenta la variación de los colores en la imagen de satélite que nos permitiera identificar la mayor concentración de bosques sobre arena blanca en una misma zona, de modo tal que optimizáramos el muestreo. Sin embargo, no podemos decir lo mismo de los varillales muy bajos (chamizales), 
ya que su pobre representación en las parcelas de muestreo (Tabla 1) puede ser un efecto de la intensidad del muestreo, pues las dos parcelas del grupo se encontraron en la misma zona.

Fue sorprendente encontrar que solo 29 especies de las 298 registradas en todo el estudio mostraron ser indicadoras de los tipos de varillales encontrados. En un escenario completamente aleatorio -sin ninguna fuerza controlando la distribución de las especies-, nosotros esperaríamos que a lo sumo 12 especies (5\% de las 249 especies que ocurrieron al menos en dos parcelas), resultaran como especies indicadoras dentro de los cinco grupos definidos. Sin embargo, al obtener 29 especies podemos afirmar que realmente existen variables ambientales que están controlando las preferencias ecológicas de estas especies, y por lo tanto son buenos indicadores de los grupos formados. Estas especies, junto a las variables ambientales estudiadas, pueden ser usadas para distinguir los tipos de varillales entre sí.

Observaciones previas han mencionado a algunas especies (p.ej. Mauritia carana, Euterpe catinga, Caraipa utilis), como representativas de algunos tipos de varillales. Sin embargo, hasta el presente estudio no se había estudiado a la comunidad entera -la variación al interior de los bosques sobre arena blanca-, y cómo estas especies pudieran servir como resúmenes ambientales, especies indicadoras, de los tipos de varillales. La ausencia de estas especies como indicadoras de los tipos de varillales observados puede ser indicativo de una gran variabilidad en la composición de las especies encontradas dentro de los varillales húmedos. Por ejemplo, las especies indicadoras del grupo 5 (chamizal), ocurren en un rango amplio de varillales húmedos, pero es en estas condiciones especialmente hostiles donde presentan una explosión en su abundancia que les permite ser identificados como especies indicadoras.

Aunque hay especies que solo se encuentran en los bosques sobre arena blanca con mal drenaje (p.ej. Euterpe catinga, Caraipa utilis, Pachira brevipes, Mauritia carana), donde a veces llegan a ser monodominantes, muy pocas especies del total parecen restringidas a los varillales húmedos. En esta dirección hay que notar que varias especies de este grupo ocurren también en otros tipos de hábitat con mal drenaje (p.ej. Rapatea ulei, Virola pavonis, Mauritia flexuosa, etc.), y al parecer llegan a estar presentes en estos varillales por el rango más amplio de tolerancia a las condiciones anóxicas que estos hábitats presentan en comparación con otras especies. Virola pavonis, la especie indicadora del grupo varillal bajo húmedo libre, es un buen ejemplo de esto. Esta especie tiene una distribución generalizada y se encuentra no solo en bosques sobre arena blanca, sino también en otros hábitats mal drenados (p.ej. pantanos de aguajales dominados por el aguaje Mauritia flexuosa, Arecaceae). Sin embargo, su abundancia y frecuencia de aparición en los varillales de dosel bajo, con mal drenaje y sotobosque relativamente libre permite que esta especie pueda ser utilizada como indicadora de estas condiciones ambientales (Tabla 1). Algo similar sucede con la especie Anacardium giganteum (Anacardiaceae), en el grupo 5 (chamizal). La abundancia y frecuencia de esta especie en este grupo le permite obtener un valor indicador (VI) dentro del rango aceptado como especie indicadora ( $p=0.0432$, Tabla 2$)$. Sin embargo, debemos notar que esta especie llega a ser un macizo árbol de dosel en los varillales húmedos, lo que no sucede en los chamizales. Un fenómeno opuesto es lo que al parecer sucede en los varillales secos, donde las especies parecen estar más restringidas a los suelos de arena blanca. Ciertamente necesitamos más investigación sobre los patrones de preferencias ecológicas de las especies dentro de los varillales.

El grupo formado por las parcelas 31 y 33 (chamizal, Tabla 1), se manifestó claramente diferente de los otros grupos, tanto fisonómicamente en el campo (baja altura, elevada densidad de tallos, cf. Tabla 3), como en el análisis de agrupamiento (Figura 1). Además, sus especies indicadoras parecen consistentes con varillales similares observados por R. García en Jenaro Herrera (río Ucayali), y pueden ser usadas para diferenciarlos de otros tipos (p.ej. la orquídea Epistephium parviflorum y la Melastomataceae Graffenrieda limbata). Estos varillales al parecer se han formado en depresiones de pantanos sobre sustrato arenoso y teniendo como dominantes del dosel, en forma dispersa, a las palmeras Mauritia flexuosa (aguaje) y Mauritiella aculeata (aguajillo).

El porcentaje de cobertura por las especies herbáceas en el piso del bosque de los varillales es muy variable (Tabla 3). Sin embargo, podemos identificar a Neoregelia sp. 1 (Bromeliaceae), Guzmania lingulata (Bromeliaceae), Anthurium atropurpureum (Araceae), y Metaxia rostrata (Pterydophyta) como relativamente frecuentes en los varillales altos secos y bajos secos. La densidad de estas especies aparentemente puede cambiar rápidamente en espacios muy cortos y ser reemplazados entre sí de la misma manera. Aunque varias especies 
del helecho Trichomanes (Pterydophyta), pueden estar presentes en un rango muy amplio de varillales, su abundancia es más notoria en los varillales mal drenados y particularmente en aquellos muy bajos (chamizales), formando verdaderos colchones que tapizan la gruesa capa de materia orgánica. Esto es particularmente notable con Trichomanes martiusii, el cual llega a ser considerado como indicadora de este grupo (Tabla 1).

En el contexto de nuestro estudio, y utilizando la terminología regional existente, los resultados presentados apoyan la separación de cinco grupos de varillales definidos por la estructura del bosque, altura del dosel y calidad del drenaje: varillal alto seco, varillal alto húmedo, varillal bajo seco, varillal bajo húmedo y chamizal húmedo.

Por último, clasificar la vegetación para comprenderla mejor es una herramienta útil para el manejo de los recursos y de esta manera para la conservación. Sin embargo, no debemos olvidar que cualquier intento humano de clasificar la naturaleza no es más que un enfoque artificial que siempre involucra decisiones arbitrarias sobre cuántas clases identificar. Esto es más crítico cuando tratamos de poner límites a la vegetación que cambia gradualmente a lo largo de gradientes ambientales. Creemos que esta clasificación de varillales que presentamos es sólo una aproximación a las complejas interacciones que ocurren en este tipo de formación vegetal y se necesitan más estudios para confirmar, mejorar o rechazar nuestros hallazgos. Sin embargo, el uso de características fáciles de percibir en el campo, utilizadas en esta clasificación, hace posible que pueda ser usada por un amplio número de usuarios.

\section{AGRADECIMIENTOS}

Queremos expresar nuestro reconocimiento a Francisco Curmayari, Samuel Coriat, Juan Díaz Alván, Willy Flores, Víctor Hugo Linares, Segundo Lúcar Oliveira, Francisco Pezo García y Marcos Ríos Paredes por colaborar con nosotros en varias fases del trabajo de campo. Silvia Flores Vásquez y José López Sánchez ayudaron en el ingreso de los datos de campo a la computadora. Agradecemos las facilidades brindadas por la jefatura de la Zona Reservada Allpahuayo-Mishana para el acceso a la ZRAM y al personal del Herbario Amazonense (AMAZ) por permitirnos consultar las colecciones. Rodolfo Vásquez Martínez revisó amablemente algunas muestras colectadas y nos dio valiosas sugerencias para el desarrollo del estudio. Paul Fine hizo accesible a nosotros bibliografía especializada en bosques sobre arena blanca. Kember Mejía, Sandra Ríos y José Álvarez Alonso dieron comentarios para mejorar la redacción del manuscrito. Finalmente, agradecemos a Illich Arista por las facilidades brindadas a lo largo del trabajo y en especial a Kalle Ruokolainen por asistirnos en el diseño del trabajo de campo y en el análisis estadístico de los datos. Este estudio fue posible por fondos del Convenio Perú - Finlandia, proyecto Diversidad Biológica de la Amazonía Peruana, BIODAMAZ.

\section{BIBLIOGRAFÍA}

ÁlVAREZ, J. 2002. Characteristic Avifauna of White-sand Forests in Northern Peruvian Amazonia. Tesis de maestría. Universidad Estatal de Louisiana, Baton Rouge, Louisiana, EE.UU.

ANDERSON, A.B. 1981. White sand vegetation of Brazilian Amazonia. Biotropica 13(3): 199-210.

ANDERSON, A.B.; PRANCE, G.T.; de Alburquerque, B.W.P. 1975. Estudos sobre a vegetação das campinas amazonicas III. Acta Amazonica 5: 225-246.

COOMES, D.A. 1997. Nutrient status of Amazonian caatinga forests in a seasonally dry area: nutrient fluxes in litter fall and analyses of soils. Can. J. For. Res. 27: 831-839.

COOMES, D.A.; GRUBB, P.J. 1996. Amazonian caatinga and related communities at La Esmeralda, Venezuela: forest structure, physiognomy and floristic, and control by soil factors. Vegetatio 122: 167-191. 
DUFRÊNE, M.; LEGENDRE, P. 1997. Species assemblages and indicator species: the need for a flexible asymmetrical approach. Ecological Monographs 67: 345-366.

DUIVENVOORDEN, J.F. 1996. Patterns of tree species richness in rain forests of the middle Caquetá area, Colombia, NW Amazonia. Biotropica 28(2): 142-158.

DUIVENVOORDEN, J.F.; LIPS, J.M. 1995. A land-ecological study of soils, vegetation and plant diversity in Colombian Amazonia. Tropembos Series 12. 438 pp.

ENCARNACIÓN, F. 1985. Introducción a la flora y vegetación de la Amazonía peruana: estado actual de los estudios, medio natural y ensayo de claves de determinación de las formaciones vegetales en la llanura Amazónica. Candollea 40: 237-252.

ENCARNACIÓN, F. 1993. El bosque y las formaciones vegetales en la llanura amazónica del Perú. Alma Mater 6: 95-114.

GENTRY, A.H. 1988. Changes in plant community diversity and floristic composition on environmental and geographical gradients. Ann. Mo. Bot. Gard. 75: 1-34.

IIAP, 2000. Informe final de la comisión técnica para la categorización y delimitación definitiva de la Zona Reservada Allpahuayo-Mishana. Instituto de Investigaciones de la Amazonía Peruana (IIAP). Informe Técnico. Iquitos, Perú. 105 pp.

JUDD, W.S.; CAMPBELL, C.S.; KELLONGG, E.A.; STEVENS, P.F. 1999. Plant Systematics: A phylogenetic approach. Sinauer Associates, Inc. Sunderland, Massachusetts. USA. 464 pp.

KALLIOLA, R.; RUOKOLAINEN, K.; TUOMISTO, H.; LINNA, A.; MÄKI, S. 1998. Mapa geoecológico de la zona de Iquitos y variación ambiental. En: Kalliola, R.; Flores Paitán, S. (eds.). 1998. Geoecología y desarrollo amazónico: estudio integrado en la zona de Iquitos, Perú. Annales Universitatis Turkuensis Ser. A II 114. 544 pp.

KAUFFMAN, S.; PAREDES, G.; MARQUINA, R. 1998. Suelos de la zona de Iquitos. En: Kalliola, R.; Flores Paitán, S. (eds.). Geoecología y desarrollo amazónico: estudio integrado en la zona de Iquitos, Perú. Annales Universitatis Turkuensis Ser. A II 114: 139-229.

KLINGE, H.; MEDINA, E.; HERRERA, R. 1977. Ecology of Amazonas caatinga forest in southern Venezuela. Acta Cient. Venez. 28: 270-276.

LEGENDRE, P.; LEGENDRE, L. 1998. Numerical Ecology. Second English Edition. Elsevier, Amsterdam.

MARENGO, J.A. 1998. Climatología de la Zona de Iquitos, Perú. En: Kalliola, R.; Flores Paitán, S. (eds.). Geoecología y desarrollo amazónico: estudio integrado en la zona de Iquitos, Peru. Annales Universitatis Turkuensis Ser A II 114: 35-57.

MCCUNE, B.; MEFFORD, M.J. 1999. PC-ORD. Multivariate Analysis of Ecological Data, version 4. MjM Software Design. 237 pp.

PROCTOR, J. 1998. Heath forests and acid soils. Bot. J. Scotl. 51(1). 1-14.

REVILLA, J.C. 1974. Descripción de los tipos de vegetación en Mishana, Río Nanay, Loreto, Perú. PAHO project AMOR-0719 report. Pan American Health Organization, Washington D.C. 
RODRÍGUEZ, W.A. 1961. Aspectos fitossociologicos das catingas do Rio Negro. Bolm. Mus. Para. "E. Goeldi”. Bot. 15: 1-41 pp.

RUOKOLAINEN, K.; TUOMISTO, H. 1998. Vegetación natural de la zona de Iquitos. En: Kalliola, R.; Flores Paitán, S. (eds.). Geoecología y desarrollo amazónico: estudio integrado en la zona de Iquitos, Perú. Annales Universitatis Turkuensis Ser. A II 114: 253-365.

TAKEUCHI, M. 1962. The structure of the Amazonian vegetation IV. High campina forest in the Upper Río Negro. J. Fac. Sci. Univ. Tokio, Sect III, Bot. 8, 279-288.

TER STEEGE, H.; JETTEN, V.G.; POLAK, A.M.; WERGER, M.J.A. 1993. Tropical rain forest types and soil factors in a watershed area in Guyana. Journal of Vegetation Science 4: 705-716.

TUOMISTO, H.; RUOKOLAINEN, K. 1994. Distribution of Pteridophyta and Melastomataceae along an edaphic gradient in an Amazonian rain forest. Journal of Vegetation Science 5(1): 25-34.

VAN DER WERFF, H. 1992. Substrate preference of Lauraceae and ferns in the Iquitos Area, Perú. Candollea 47: 11-20.

VITOUSEK, P.M.; SANFORD, R.L. 1986. Nutrient cycling in moist tropical forest. Ann. Rev. Ecol. Syst. 17: 137-167.

VORMISTO, J.; PHILLIPS, O.L.; RUOKOLAINEN, K.; TUOMISTO, H.; VÁSQUEZ, R. 2000. A comparison of small-scale distribution patterns of four plant groups in an Amazonian rainforest. Ecography 23: 349-359. 
Tabla 2. Lista de especies encontradas en el estudio y resultados de la prueba de Monte Carlo en el análisis de especies indicadoras (ver texto). Las familias de helechos fueron agrupadas en el orden Pterydophyta. Las letras dentro de paréntesis en la columna familia representan los acrónimos del nombre antiguo al que estaba asignado la familia (cf. Judd et al., 1999), a excepción de la familia Fabaceae, que representan subfamilias. Las especies con la p no mayor de 0.05 (especies indicadoras) están resaltadas en negrita.

\begin{tabular}{|c|c|c|c|c|c|}
\hline Familia & Género & Especie & Autor & $\mathrm{p}^{1}$ & $\begin{array}{c}\text { Grupo } \\
\text { máx. }\end{array}$ \\
\hline Anacardiaceae & Anacardium & giganteum & W. Hancock ex Engl. & 0.0432 & 5 \\
\hline Anacardiaceae & Tapirira & guianensis & Aubl. & 0.3460 & 3 \\
\hline Anacardiaceae & Tapirira & retusa & Ducke & 0.7160 & 3 \\
\hline Anacardiaceae & Thyrsodium & spruceanum & Benth. & 0.8964 & 4 \\
\hline Annonaceae & Anaxagorea & brevipes & Benth. & 0.1178 & 4 \\
\hline Annonaceae & Anaxagorea & manausensis & Timmerman & 0.2494 & 4 \\
\hline Annonaceae & Annonaceae & sp. 1 & & 1.0000 & 4 \\
\hline Annonaceae & Annonaceae & sp. 3 & & 0.0680 & 5 \\
\hline Annonaceae & Annonaceae & sp. 4 & & 1.0000 & 3 \\
\hline Annonaceae & Annonaceae & sp. 5 & & 1.0000 & 4 \\
\hline Annonaceae & Annonaceae & sp. 6 & & 0.5718 & 4 \\
\hline Annonaceae & Annonaceae & sp.11 & & 0.7517 & 4 \\
\hline Annonaceae & Diclinanona & tessmannii & Diels & 0.0975 & 4 \\
\hline Annonaceae & Duguetia & latifolia & R. E. Fr. & 0.4896 & 4 \\
\hline Annonaceae & Guatteria & decurrens & R. E. Fr. & 0.8598 & 4 \\
\hline Annonaceae & Guatteria & megalophylla & Diels & 0.2269 & 3 \\
\hline Annonaceae & Guatteriopsis & sessiliflora & (Bentham) Fries, R.E. & 0.1362 & 3 \\
\hline Annonaceae & Oxandra & euneura & Diels & 0.0372 & 4 \\
\hline Annonaceae & Pseudoxandra & sp. 1 & & 1.0000 & 3 \\
\hline Annonaceae & Ruizodendron & ovale & (Ruiz \& Pav.) R. E. Fr. & 1.0000 & 3 \\
\hline Annonaceae & Tetrameranthus & pachycarpus & Westra & 0.3150 & 4 \\
\hline Annonaceae & Xylopia & benthamii & R. E. Fr. & 0.1206 & 3 \\
\hline Annonaceae & Xylopia & parviflora & Spruce & 0.8966 & 4 \\
\hline Apocynaceae & Apocynaceae & sp. 1 & & 0.5719 & 4 \\
\hline Apocynaceae & Aspidosperma & excelsum & Benth. & 0.6941 & 1 \\
\hline Apocynaceae & Aspidosperma & pichonianum & Woodson & 0.0204 & 4 \\
\hline Apocynaceae & Aspidosperma & schultesii & Woodson & 0.0884 & 4 \\
\hline Apocynaceae & Lacmellea & klugii & Monach. & 0.0548 & 4 \\
\hline Apocynaceae & Macoubea & guianensis & Aubl. & 0.4094 & 3 \\
\hline Aquifoliaceae & Ilex & sp. 1 & & 0.6473 & 5 \\
\hline Aquifoliaceae & Ilex & sp. 2 & & 0.9856 & 3 \\
\hline Araceae & Anthurium & $\begin{array}{l}\text { atropurpureum var } \\
\text { atropurpureum }\end{array}$ & R.E. Schult. \& Schum. & 0.0957 & 4 \\
\hline Apiaceae (Aral) & Dendropanax & umbellatus & $\begin{array}{l}\text { (Ruiz \& Pav.) Decne. \& } \\
\text { Planch. }\end{array}$ & 0.0044 & 5 \\
\hline Apiaceae (Aral) & Schefflera & morototoni & $\begin{array}{l}\text { (Aubl.) Maguire, Steyerm. } \\
\& \text { Frodin }\end{array}$ & 0.2236 & 4 \\
\hline Arecaceae & Mauritiella & aculeata & Kunth & 1.0000 & 3 \\
\hline Arecaceae & Euterpe & catinga & Wallace & 0.2881 & 1 \\
\hline Arecaceae & Mauritia & carana & Wallace & 0.2412 & 1 \\
\hline Arecaceae & Mauritia & flexuosa & L. f. & 0.0864 & 5 \\
\hline Bignoniaceae & Jacaranda & macrocarpa & Bureau \& K. Schum. & 0.1095 & 4 \\
\hline Bignoniaceae & Tabebuia & incana & A. G. Gentry & 0.0907 & 2 \\
\hline Boraginaceae & Cordia & nodosa & & 1.0000 & 3 \\
\hline Bromeliaceae & Guzmania & lingulata & (L.) $\mathrm{Mez}$ & 0.1392 & 2 \\
\hline Bromeliaceae & Neoregelia & sp. 1 & & 0.4535 & 3 \\
\hline
\end{tabular}




\begin{tabular}{|c|c|c|c|c|c|}
\hline Familia & Género & Especie & Autor & $\mathrm{p}^{1}$ & $\begin{array}{c}\text { Grupo } \\
\text { máx. }^{2}\end{array}$ \\
\hline Burseraceae & Protium & aracouchini & (Aubl.) Marchand & 0.7584 & 4 \\
\hline Burseraceae & Protium & ferrugineum & (Engl.) Engl. & 0.3258 & 4 \\
\hline Burseraceae & Protium & paniculatum & Engl. & 0.7525 & 4 \\
\hline Burseraceae & Protium & subserratum & (Engl.) Engl. & 0.1437 & 4 \\
\hline Capparidaceae & Capparis & sola & J. F. Macbr. & 0.5682 & 4 \\
\hline Caryocariaceae & Anthodiscus & pilosus & Ducke & 0.5653 & 4 \\
\hline Cecropiaceae & Pourouma & tomentosa & Mart. ex Miq. & 0.2348 & 3 \\
\hline Celastraceae & Maytenus & amazonica & Mart. & 0.3128 & 4 \\
\hline Celastraceae & Maytenus & macrocarpa & (Ruiz \& Pav.) Briq. & 0.5700 & 4 \\
\hline Clusiaceae & Calophyllum & brasiliensis & Cambess. & 0.5506 & 5 \\
\hline Clusiaceae & Caraipa & tereticaulis & Tul. & 0.7215 & 1 \\
\hline Clusiaceae & Caraipa & utilis & Vásquez & 0.1097 & 3 \\
\hline Clusiaceae & Clusia & amazonica & Planch. \& Triana & 0.2016 & 2 \\
\hline Clusiaceae & Clusia & sp. 1 & & 0.2611 & 2 \\
\hline Clusiaceae & Garcinia & madruno & (Kunth) Hammel & 0.1457 & 4 \\
\hline Clusiaceae & Haploclathra & cordata & Vásquez & 0.7652 & 3 \\
\hline Clusiaceae & Haploclathra & paniculata & (Mart.) Benth. & 0.5700 & 4 \\
\hline Clusiaceae & Tovomita & calophyllophylla & & 0.0922 & 4 \\
\hline Clusiaceae & Tovomita & cephalostigma & Vesque & 0.1446 & 4 \\
\hline Clusiaceae & Tovomita & sp. 1 & & 1.0000 & 3 \\
\hline Clusiaceae & Tovomita & sp. 2 & & 0.4170 & 4 \\
\hline Clusiaceae & Tovomita & umbellata & Benth. & 0.1167 & 4 \\
\hline Combretaceae & Buchenavia & amazonia & Alvan \& Stace & 0.0829 & 1 \\
\hline Combretaceae & Buchenavia & grandis & Ducke & 0.5665 & 4 \\
\hline Combretaceae & Buchenavia & reticulata & Eichler & 0.0368 & 4 \\
\hline Costaceae & Costus & lasius & Loes & 0.8685 & 4 \\
\hline Chrysobalanaceae & Couepia & parillo & DC. & 0.0033 & 4 \\
\hline Chrysobalanaceae & Couepia & williamsii & J. F. Macbr. & 0.3245 & 3 \\
\hline Chrysobalanaceae & Licania & heteromorpha & Benth. & 0.5601 & 4 \\
\hline Chrysobalanaceae & Licania & intrapetiolaris & Spruce ex Hook. f. & 0.2629 & 3 \\
\hline Chrysobalanaceae & Licania & micrantha & Miq. & 1.0000 & 3 \\
\hline Chrysobalanaceae & Licania & octandra & $\begin{array}{l}\text { (Hoffmanns. ex Roem. \& } \\
\text { Shult) Kuntze }\end{array}$ & 0.2852 & 2 \\
\hline Dichapetalaceae & Tapura & amazonica & Poepp. \& Endl. & 0.6407 & 3 \\
\hline Dilleniaceae & Doliocarpus & dentathus & (Aubl.) Standl. & 0.0128 & 5 \\
\hline Dioscoreaceae & Dioscorea & laxiflora & Mart. ex Griseb. & 0.2801 & 5 \\
\hline Ebenaceae & Diospyros & egleri & Pires \& Cav. & 0.5682 & 4 \\
\hline Ebenaceae & Diospyros & tessmannii & Mildbr. & 0.1474 & 4 \\
\hline Elaeocarpaceae & Sloanea & floribunda & Spruce ex Benth. & 0.2016 & 1 \\
\hline Elaeocarpaceae & Sloanea & latifolia & (Rich.) K. Schum. & 0.6821 & 2 \\
\hline Elaeocarpaceae & Sloanea & sp. 1 & & 0.1585 & 4 \\
\hline Elaeocarpaceae & Sloanea & spathulata & Earle Sm. & 0.0204 & 5 \\
\hline Erythroxylaceae & Erythroxylum & citrifolium & A. St.-Hil. & 1.0000 & 3 \\
\hline Euphorbiaceae & Aparisthmium & cordatum & (A. Juss.) Baill. & 0.0093 & 4 \\
\hline Euphorbiaceae & Hevea & guianensis & Aubl. & 0.0186 & 5 \\
\hline Euphorbiaceae & Mabea & subsessilis & Pax \& K. Hoffm. & 0.0500 & 4 \\
\hline Euphorbiaceae & Micrandra & elata & Mull. Arg. & 0.1489 & 4 \\
\hline Euphorbiaceae & Pausandra & martinii & Baill. & 0.0245 & 4 \\
\hline Euphorbiaceae & Pera & citriodora & Baill. & 0.2305 & 2 \\
\hline Fabaceae (Caes) & Dicymbe & uaiparuensis & R. S. Cowan & 0.0207 & 2 \\
\hline Fabaceae (Caes) & Fabaceae (Caes) & sp. 2 & & 0.5718 & 4 \\
\hline Fabaceae (Caes) & Fabaceae (Caes) & sp. 1 & & 1.0000 & 3 \\
\hline Fabaceae (Caes) & Fabaceae (Caes) & sp. 3 & & 0.1074 & 3 \\
\hline
\end{tabular}




\begin{tabular}{|c|c|c|c|c|c|}
\hline Familia & Género & Especie & Autor & $\mathrm{p}^{1}$ & $\begin{array}{c}\text { Grupo } \\
\text { máx. }^{2}\end{array}$ \\
\hline Fabaceae (Caes) & Macrolobium & bifolium & (Aubl.) Pers. & 0.1798 & 4 \\
\hline Fabaceae (Caes) & Macrolobium & limbatum & Spruce ex Benth. & 0.2215 & 4 \\
\hline Fabaceae (Caes) & Macrolobium & microcalyx & Ducke & 0.0541 & 5 \\
\hline Fabaceae (Caes) & Macrolobium & sp. 1 & & 0.7020 & 3 \\
\hline Fabaceae (Caes) & Pentaclethra & macroloba & (Willd.) Kuntze & 0.5042 & 3 \\
\hline Fabaceae (Caes) & Tachigali & bracteosa & (Harms) Zarucchi \& Pipoly & 0.1184 & 4 \\
\hline Fabaceae (Caes) & Tachigali & paniculata & Aubl. & 0.1463 & 4 \\
\hline Fabaceae (Caes) & Tachigali & ptychophysca & Spruce ex Benth. & 0.0789 & 4 \\
\hline Fabaceae (Caes) & Tachigali & sp. 1 & & 0.5601 & 4 \\
\hline Fabaceae (Caes) & Taralea & oppositifolia & Aubl. & 0.1209 & 4 \\
\hline Fabaceae (Mimo) & Abarema & adenophora & $\begin{array}{l}\text { (Ducke) Barneby \& } \\
\text { J. M. Grimes }\end{array}$ & 0.5700 & 4 \\
\hline Fabaceae (Mimo) & Acacia & sp. 1 & & 0.1085 & 3 \\
\hline Fabaceae (Mimo) & Inga & sp. 1 & & 0.1604 & 3 \\
\hline Fabaceae (Mimo) & Inga & sp. 2 & & 0.3008 & 4 \\
\hline Fabaceae (Mimo) & Inga & sp. 3 & & 0.5718 & 4 \\
\hline Fabaceae (Mimo) & Inga & sp. 4 & & 1.0000 & 3 \\
\hline Fabaceae (Mimo) & Inga & sp. 5 & & 0.3836 & 2 \\
\hline Fabaceae (Mimo) & Parkia & igneiflora & Ducke & 0.7724 & 3 \\
\hline Fabaceae (Mimo) & Pithecellobium & auriculatum & Benth. & 0.1304 & 1 \\
\hline Fabaceae (Papi) & Fabaceae (Papi) & sp. 1 & & 0.5008 & 3 \\
\hline Fabaceae (Papi) & Fabaceae (Papi) & sp. 2 & & 0.1122 & 1 \\
\hline Fabaceae (Papi) & Fabaceae (Papi) & sp. 3 & & 1.0000 & 3 \\
\hline Fabaceae (Papi) & Fabaceae (Papi) & sp. 4 & & 1.0000 & 3 \\
\hline Fabaceae (Papi) & Fabaceae (Papi) & sp. 5 & & 0.6164 & 3 \\
\hline Fabaceae (Papi) & Hymenolobium & nitidum & Benth. & 0.1308 & 3 \\
\hline Fabaceae (Papi) & Pterocarpus & rohrii & Vahl & 0.5665 & 4 \\
\hline Fabaceae (Papi) & Swartzia & benthamiana & Miq. & 0.2099 & 4 \\
\hline Fabaceae (Papi) & Swartzia & cardiosperma & Spruce ex Benth. & 0.3957 & 4 \\
\hline Fabaceae (Papi) & Swartzia & pendula & Spruce ex Benth. & 0.0640 & 4 \\
\hline Fabaceae (Papi) & Swartzia & polyphylla & DC. & 0.3131 & 4 \\
\hline Fabaceae (Papi) & Swartzia & sp. 6 & & 1.0000 & 3 \\
\hline Fabaceae (Papi) & Swartzia & tessmannii & Harms & 0.5279 & 3 \\
\hline Flacourtiaceae & Casearia & sp. 1 & & 1.0000 & 3 \\
\hline Flacourtiaceae & Ryania & speciosa & Vahl & 0.0002 & 4 \\
\hline Gentinaceae & Tachia & occidentalis & & 0.1952 & 3 \\
\hline Humiriaceae & Humiria & balsamifera & Aubl. & 0.3237 & 4 \\
\hline Humiriaceae & Humiriaceae & sp. 1 & & 0.2359 & 3 \\
\hline Humiriaceae & Saccoglotis & ceratocarpa & Ducke & 0.5718 & 4 \\
\hline Humiriaceae & Sacoglottis & sp. 1 & & 0.6212 & 3 \\
\hline Icacinaceae & Discophora & guianensis & Miers & 0.1434 & 4 \\
\hline Icacinaceae & Emmotum & floribundum & R. A. Howard & 0.3249 & 2 \\
\hline Lauraceae & Aniba & megaphylla & Mez & 0.3073 & 4 \\
\hline Lauraceae & Endlicheria & citriodora & van der Werff & 0.5267 & 1 \\
\hline Lauraceae & Lauraceae & sp. 1 & & 0.1078 & 1 \\
\hline Lauraceae & Lauraceae & sp. 10 & & 0.5665 & 4 \\
\hline Lauraceae & Lauraceae & sp. 11 & & 0.2255 & 1 \\
\hline Lauraceae & Lauraceae & sp. 12 & & 0.7482 & 4 \\
\hline Lauraceae & Lauraceae & sp. 13 & & 0.8683 & 4 \\
\hline Lauraceae & Lauraceae & sp. 14 & & 0.5233 & 4 \\
\hline Lauraceae & Lauraceae & sp. 15 & & 0.6204 & 4 \\
\hline Lauraceae & Lauraceae & sp. 2 & & 0.0555 & 3 \\
\hline Lauraceae & Lauraceae & sp. 3 & & 0.3122 & 4 \\
\hline
\end{tabular}




\begin{tabular}{|c|c|c|c|c|c|}
\hline Familia & Género & Especie & Autor & $\mathrm{p}^{1}$ & $\begin{array}{l}\text { Grupo } \\
\text { máx. }^{2}\end{array}$ \\
\hline Lauraceae & Lauraceae & sp. 4 & & 0.1826 & 4 \\
\hline Lauraceae & Lauraceae & sp. 5 & & 0.5601 & 4 \\
\hline Lauraceae & Lauraceae & sp. 6 & & 0.5601 & 4 \\
\hline Lauraceae & Lauraceae & sp. 7 & & 1.0000 & 3 \\
\hline Lauraceae & Lauraceae & sp. 8 & & 0.7768 & 3 \\
\hline Lauraceae & Lauraceae & sp. 9 & & 1.0000 & 4 \\
\hline Lauraceae & Ocotea & aciphylla & (Nees) Mez & 0.0953 & 4 \\
\hline Lauraceae & Ocotea & argirophylla & Ducke & 0.4800 & 4 \\
\hline Lauraceae & Ocotea & gracilis & (Meisn.) Mez & 0.3168 & 4 \\
\hline Lauraceae & Ocotea & olivacea & A. C. Sm. & 0.1280 & 4 \\
\hline Lauraceae & Ocotea & licanioides & A. C. Sm. & 0.5381 & 4 \\
\hline Lecythidaceae & Eschweilera & sp. 1 & & 0.3210 & 4 \\
\hline Linaceae & Roucheria & punctata & (Ducke) Ducke & 0.1291 & 4 \\
\hline Linaceae & Roucheria & schomburgkii & (Planch.) Ducke & 0.1923 & 4 \\
\hline Lissocarpaceae & Lissocarpa & jensonii & Vásquez & 0.7530 & 4 \\
\hline Lissocarpaceae & Lissocarpa & stenocarpa & Steyerm. & 0.0504 & 4 \\
\hline Loganiaceae & Potalia & amara & Aubl. & 0.3437 & 1 \\
\hline Malphigiaceae & Byrsonima & stipulina & J.F. Macbr. & 0.0022 & 4 \\
\hline Melastomataceae & Adelobotrys & marginata & Brades & 0.1122 & 1 \\
\hline Melastomataceae & Clidemia & epibaterium & D. C. & 0.9455 & 2 \\
\hline Melastomataceae & Graffenrieda & limbata & Triana & 0.0015 & 5 \\
\hline Malvaceae (Tili) & Lueheopsis & althaeiflora & (Spruce ex Benth.) Burret & 0.4399 & 4 \\
\hline Melastomataceae & Miconia & sp. 1 & & 0.1407 & 2 \\
\hline Melastomataceae & Miconia & sp. 2 & & 0.5601 & 4 \\
\hline Melastomataceae & Miconia & sp. 3 & & 0.5700 & 4 \\
\hline Melastomataceae & Miconia & sp. 4 & & 0.6300 & 3 \\
\hline Malvaceae (Bomb) & Pachira & brevipes & (A. Robyns) W. S. Alverson & 0.2164 & 2 \\
\hline Meliaceae & Guarea & $\begin{array}{l}\text { pubescens subsp } \\
\text { pubescens }\end{array}$ & (Rich.) A. Juss. & 0.7784 & 4 \\
\hline Melastomataceae & Miconia & sp. 5 & & 0.5718 & 4 \\
\hline Meliaceae & Tococa & guianensis & Aubl. & 0.2464 & 1 \\
\hline Meliaceae & Guarea & cristata & T. D. Penn. & 0.0674 & 4 \\
\hline Moraceae & Brosimum & rubescens & Taub. & 0.1388 & 4 \\
\hline Moraceae & Brosimum & utile & (H.B.K.) Pittier & 0.7818 & 1 \\
\hline Moraceae & Dioscorea & laxiflora & Mart. ex Griseb. & 0.5471 & 1 \\
\hline Moraceae & Ficus & sp. 1 & & 0.0882 & 5 \\
\hline Moraceae & Trichilia & pallida & Sw. & 0.6946 & 3 \\
\hline Moraceae & Helycostilis & sp. 1 & & 0.2915 & 4 \\
\hline Myristicaceae & Iryanthera & polyneura & Ducke & 0.5708 & 4 \\
\hline Moraceae & Naucleopsis & sp. 1 & & 0.3300 & 3 \\
\hline Myristicaceae & Virola & calophylla & (Spruce) Warb. & 0.5601 & 4 \\
\hline Myristicaceae & Virola & cf surinamensis & (Rol. ex Rotlb.) Warb. & 0.2723 & 2 \\
\hline Myristicaceae & Virola & pavonis & (A. DC.) A. C. Sm. & 0.0007 & 1 \\
\hline Myrsinaceae & Cybianthus & peruvianus & (A. DC.) Miq. & 0.3707 & 4 \\
\hline Myrsinaceae & Cybianthus & resinosus & $\mathrm{Mez}$ & 0.5026 & 3 \\
\hline Myrsinaceae & Cybianthus & sp. 1 & & 0.1227 & 3 \\
\hline Myrsinaceae & Cybianthus & spichigeri & Pipoly & 1.0000 & 3 \\
\hline Myristicaceae & Iryanthera & Ulei & Warb. & 0.2825 & 3 \\
\hline Myrtaceae & Eugenia & sp. 1 & & 0.2663 & 2 \\
\hline Myrtaceae & Marliera & caudata & & 0.4363 & 3 \\
\hline Myrtaceae & Myrcia & sp. 1 & & 0.0846 & 4 \\
\hline Myrtaceae & Myrcia & sp. 2 & & 0.0678 & 4 \\
\hline Myrtaceae & Myrcia & sp. 3 & & 0.4146 & 4 \\
\hline
\end{tabular}




\begin{tabular}{|c|c|c|c|c|c|}
\hline Familia & Género & Especie & Autor & $\mathrm{p}^{1}$ & $\begin{array}{c}\text { Grupo } \\
\text { máx. }\end{array}$ \\
\hline Myrtaceae & Myrtaceae & sp. 1 & & 0.0740 & 4 \\
\hline Myrtaceae & Myrtaceae & sp. 10 & & 0.2774 & 2 \\
\hline Myrtaceae & Myrtaceae & sp. 12 & & 0.2293 & 1 \\
\hline Myrtaceae & Myrtaceae & sp. 13 & & 0.8726 & 4 \\
\hline Myrtaceae & Myrtaceae & sp. 16 & & 0.6330 & 3 \\
\hline Myrtaceae & Myrtaceae & sp. 17 & & 0.2723 & 2 \\
\hline Myrtaceae & Myrtaceae & sp. 2 & & 0.0471 & 4 \\
\hline Rutaceae & Ravenia & biramosa & Ducke & 0.0436 & 4 \\
\hline Myrtaceae & Myrtaceae & sp. 6 & & 0.8589 & 4 \\
\hline Myrtaceae & Myrtaceae & sp. 8 & & 0.3147 & 4 \\
\hline Myrtaceae & Myrtaceae & sp. 9 & & 0.7513 & 4 \\
\hline Nyctaginaceae & Neea & divaricata & Poepp. \& Endl. & 0.0117 & 5 \\
\hline Nyctaginaceae & Neea & macrophylla & Poepp. \& Endl. & 0.2559 & 4 \\
\hline Nyctaginaceae & Neea & parviflora & Poepp. \& Endl. & 1.0000 & 3 \\
\hline Nyctaginaceae & Neea & sp. 1 & & 0.1624 & 4 \\
\hline Nyctaginaceae & Neea & verticillata & Ruíz \& Pav. & 0.1394 & 4 \\
\hline Ochnaceae & Ouratea & sp. 1 & & 0.8502 & 3 \\
\hline Ochnaceae & Ouratea & sp. 3 & & 0.2361 & 4 \\
\hline Ochnaceae & Ouratea & sp. 2 & & 0.1718 & 3 \\
\hline Olacaceae & Dulacia & candida & (Poepp.) Kuntze & 0.3812 & 3 \\
\hline Olacaceae & Heisteria & insculpta & Sleumer & 0.3159 & 4 \\
\hline Opiliaceae & Agonandra & sp. 1 & & 0.3894 & 4 \\
\hline Orchidaceae & Epistephium & parviflorum & Hook. f. & 0.0053 & 5 \\
\hline Passifloraceae & Dilkea & retusa & Mast. & 0.0549 & 4 \\
\hline Picramniaceae & Picramnia & sp. 1 & & 0.1756 & 4 \\
\hline Piperaceae & Piper & mituense & Trel. \& Yunck. & 0.5718 & 4 \\
\hline Piperaceae & Piper & sp. 1 & & 0.3369 & 4 \\
\hline Polygonaceae & Coccoloba & sp. 1 & & 0.6833 & 4 \\
\hline Pteridophyta & Cyathea & sp. 1 & & 1.0000 & 3 \\
\hline Pterydophyta & Elaphoglossum & discolor & (Kuhn.) C.Chr. & 0.0989 & 2 \\
\hline Pterydophyta & Metaxya & lanosa & A. R. Smith \& H. Tuomisto & 0.5186 & 2 \\
\hline Pterydophyta & Pterydophyta & sp. 1 & & 0.0729 & 2 \\
\hline Pterydophyta & Saccoloma & inaequale & (Kunze) Mett. & 0.2938 & 4 \\
\hline Pterydophyta & Trichomanes & bicorne & Hook. & 0.1835 & 1 \\
\hline Pterydophyta & Trichomanes & martiusii & C. Presl & 0.0395 & 5 \\
\hline Rapateaceae & Rapatea & ulei & Pilg. & 0.8106 & 3 \\
\hline Rhizophoraceae & Sterigmapetalum & obovatum & & 0.6102 & 3 \\
\hline Rubiaceae & Amaioua & guianensis & Aubl. & 1.0000 & 4 \\
\hline Rubiaceae & Ferdinandusa & chloranta & (Wedd.) Standl. & 0.5238 & 1 \\
\hline Rubiaceae & Ixora & Ulei & K. Krause & 0.5682 & 4 \\
\hline Rubiaceae & Ladenbergia & sp. 1 & & 0.1299 & 3 \\
\hline Rubiaceae & Pagamea & coriacea & Spruce ex Benth. & 0.2401 & 4 \\
\hline Rubiaceae & Pagamea & guianensis & Aubl. & 0.0860 & 3 \\
\hline Rubiaceae & Palicourea & condensata & Standl. & 1.0000 & 3 \\
\hline Rubiaceae & Palicourea & sp. 1 & & 0.5306 & 3 \\
\hline Rubiaceae & Kutchubaea & cf. sericantha & Standl. & 0.7541 & 3 \\
\hline Rosaceae & Prunus & detrita & J. F. Macbr. & 0.1659 & 4 \\
\hline Rubiaceae & Psychotria & axyllaris & Willd. & 0.3913 & 2 \\
\hline Rubiaceae & Psychotria & sp. 1 & & 0.8652 & 4 \\
\hline Rubiaceae & Psychotria & sp. 2 & & 0.1939 & 3 \\
\hline Rubiaceae & Psychotria & sp. 3 & & 0.3101 & 1 \\
\hline Rubiaceae & Psychotria & sp. 4 & & 0.0012 & 5 \\
\hline Rubiaceae & Remigia & ulei & K. Krause & 0.0176 & 5 \\
\hline
\end{tabular}




\begin{tabular}{|c|c|c|c|c|c|}
\hline Familia & Género & Especie & Autor & $\mathrm{p}^{1}$ & $\begin{array}{c}\begin{array}{c}\text { Grupo } \\
\text { máx. }^{2}\end{array} \\
\end{array}$ \\
\hline Rubiaceae & Alibertia & hispida & Ducke & 0.0056 & 5 \\
\hline Rubiaceae & Rubiaceae & sp. 2 & & 0.0921 & 4 \\
\hline Rubiaceae & Rubiaceae & sp. 3 & & 0.2211 & 4 \\
\hline Rubiaceae & Rubiaceae & sp. 5 & & 0.6187 & 1 \\
\hline Rubiaceae & Rubiaceae & sp. 6 & & 0.2707 & 4 \\
\hline Rubiaceae & Rubiaceae & sp. 4 & & 0.4324 & 3 \\
\hline Rubiaceae & Rudgea & fissistipula & Mull. Arg. & 0.0989 & 1 \\
\hline Rutaceae & Adiscanthus & fusciflorus & Ducke & 0.0114 & 3 \\
\hline Sapindaceae & Cupania & hispida & Radlk. & 0.1874 & 4 \\
\hline Sapindaceae & Cupania & sp. 1 & & 0.1906 & 5 \\
\hline Sapindaceae & Cupania & sp. 2 & Vahl & 0.1876 & 3 \\
\hline Rutaceae & Leptothyrsa & sprucei & Hook. f. & 0.0905 & 4 \\
\hline Sapindaceae & Matayba & guianensis & & 1.0000 & 4 \\
\hline Sapindaceae & Matayba & inelegans & Spruce ex Radlk. & 0.2111 & 3 \\
\hline Sapindaceae & Matayba & sp. 1 & & 0.0084 & 4 \\
\hline Sapindaceae & Matayba & sp. 2 & & 0.0515 & 1 \\
\hline Sapindaceae & Matayba & sp. 3 & & 1.0000 & 3 \\
\hline Sapindaceae & Matayba & sp. 4 & & 0.0978 & 4 \\
\hline Sapindaceae & Matayba & sp. 5 & & 0.5601 & 4 \\
\hline Sapindaceae & Talisia & sp. 1 & & 1.0000 & 3 \\
\hline Sapotaceae & Chrysophyllum & bombycinum & T. D. Penn. & 0.1751 & 4 \\
\hline Sapotaceae & Chrysophyllum & manaosensis & (Aubrév.) T. D. Penn. & 0.0379 & 3 \\
\hline Sapotaceae & Chrysophyllum & prieurii & A.DC. & 0.2043 & 4 \\
\hline Sapotaceae & Chrysophyllum & sanguinolentum & (Pierre) Baehni & 0.6597 & 1 \\
\hline Sapotaceae & Manilkara & sp. 1 & & 0.3833 & 3 \\
\hline Sapotaceae & Micropholis & broquidodroma & (Mart. \& Eichler) Pierre & 1.0000 & 4 \\
\hline Sapotaceae & Micropholis & guyanensis (A.DC.) & (Baehni) T. D. Penn. & 0.1412 & 4 \\
\hline Sapotaceae & Micropholis & $\begin{array}{l}\text { Pierre subsp duckeana } \\
\text { venulosa }\end{array}$ & (Mart. \& Eichler) Pierre & 0.1077 & 4 \\
\hline Sapotaceae & Pouteria & cf reticulata & (Engl.) Eyma & 1.0000 & 3 \\
\hline Sapotaceae & Pouteria & cf rostrata & (Huber) Baehni & 0.1264 & 4 \\
\hline Sapotaceae & Pouteria & cuspidata & Woodson & 0.4538 & 5 \\
\hline Sapotaceae & Pouteria & lucumifolia & (A. DC.) Baehni & 0.2300 & 3 \\
\hline Sapotaceae & Pouteria & oblanceolata & Pires & 0.9574 & 4 \\
\hline Sapotaceae & Pouteria & sp. 1 & & 0.3039 & 4 \\
\hline Sapotaceae & Pradosia & aff cochlearia & (Lecomte) T. D. Penn. & 0.2532 & 3 \\
\hline Sapindaceae & Sapindaceae & sp. 3 & & 0.7468 & 4 \\
\hline Sapotaceae & Sapotaceae & sp. 1 & & 1.0000 & 4 \\
\hline Sapotaceae & Sapotaceae & sp. 2 & & 0.6180 & 3 \\
\hline Simaroubaceae & Simaba & polyphylla & $\begin{array}{l}\text { (Cavalcante) W. W. } \\
\text { Thomas }\end{array}$ & 0.0457 & 4 \\
\hline Simaroubaceae & Simaba & sp. 1 & & 0.4071 & 4 \\
\hline Simaroubaceae & Simarouba & amara & Aubl. & 1.0000 & 4 \\
\hline Siparunaceae & Siparuna & guianensis & Aubl. & 0.0031 & 5 \\
\hline Smilacaceae & Smilax & aff cumanensis & Willd. & 0.1839 & 4 \\
\hline Solanaceaea & Cestrum & microcalyx & Francey & 0.1023 & 4 \\
\hline Styracaceae & Styrax & sp. 1 & & 0.0258 & 3 \\
\hline $\begin{array}{l}\text { Ternstroemiaceae } \\
\text { (Thea) }\end{array}$ & Ternstroemia & klugiana & Kobuski & 0.2632 & 2 \\
\hline
\end{tabular}

${ }^{1}$ Proporción de pruebas aleatorizadas con valor indicador (VI) igual o excediendo el valor indicador observado.

${ }^{2}$ Grupo indicador para el grupo con el máximo valor indicador (VI) observado. 
Tabla 3. Características estructurales y ambientales de las parcelas estudiadas

\begin{tabular}{ccccccccc}
\hline $\begin{array}{c}\text { Número } \\
\text { parcela }\end{array}$ & $\begin{array}{c}\text { Grosor de la materia } \\
\text { orgánica }(\mathrm{cm})\end{array}$ & $\begin{array}{c}\text { Altura } \\
\text { promedio } \\
\text { del dosel } \\
(\mathrm{m})\end{array}$ & $\begin{array}{c}\text { Cobert. } \\
\text { piso del } \\
\text { bosque } \\
\%\end{array}$ & $\begin{array}{c}\text { Núm. tallos } \\
\text { clase } \\
\text { 1m alt-1 cm dap }\end{array}$ & $\begin{array}{c}\text { Núm. tallos } \\
\text { clase } \\
1-2.5 \text { dap }\end{array}$ & $\begin{array}{c}\text { Núm. tallos } \\
\text { clase } \\
2.5-5 \text { dap }\end{array}$ & $\begin{array}{c}\text { Núm. tallos } \\
\text { clase } \\
5-10 \text { dap }\end{array}$ & $\begin{array}{c}\text { Núm. total } \\
\text { tallos 1-10 } \\
\text { dap } \\
\text { p/parcela }\end{array}$ \\
\hline 1 & 33.8 & 18 & 7.707 & 480 & 83 & 71 & 40 & 674 \\
2 & 8.08 & 7.2 & 64.769 & 885 & 224 & 136 & 79 & 1324 \\
3 & 11.5 & 14 & 22.076 & 497 & 207 & 111 & 55 & 870 \\
4 & 19.4 & 21 & 7.611 & 420 & 183 & 62 & 25 & 690 \\
5 & 26.4 & 13.5 & 7.232 & 414 & 132 & 152 & 81 & 779 \\
6 & 18.8 & 18 & 21.4 & 1409 & 456 & 268 & 59 & 2192 \\
7 & 17.4 & 20 & 43.132 & 633 & 261 & 170 & 49 & 1113 \\
8 & 14.6 & 15 & 14.814 & 830 & 346 & 153 & 40 & 1369 \\
9 & 12.4 & 19 & 41.52 & 504 & 216 & 123 & 46 & 889 \\
10 & 12.2 & 26 & 4.094 & 504 & 218 & 133 & 59 & 914 \\
11 & 3.8 & 14 & 59.44 & 527 & 176 & 115 & 75 & 893 \\
12 & 1.7 & 8 & 80.9 & 827 & 313 & 217 & 123 & 1480 \\
13 & 1.4 & 17 & 83.97 & 232 & 104 & 75 & 52 & 463 \\
14 & 42.4 & 10 & 14.09 & 810 & 162 & 168 & 115 & 1255 \\
15 & 10.6 & 10 & 24.766 & 1159 & 369 & 219 & 107 & 1854 \\
16 & 2 & 16 & 75.49 & 520 & 171 & 115 & 46 & 852 \\
17 & 1 & 18 & 8.64 & 378 & 130 & 67 & 27 & 602 \\
18 & 1.4 & 13 & 36.691 & 927 & 235 & 158 & 83 & 1403 \\
19 & 2.1 & 7 & 89.446 & 266 & 94 & 89 & 70 & 519 \\
20 & 21.6 & 10 & 12.115 & 949 & 551 & 308 & 77 & 1885 \\
\hline
\end{tabular}

